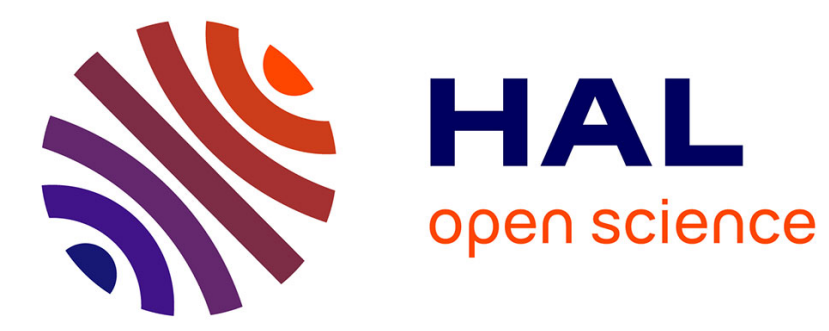

\title{
Line transversals to disjoint balls
}

Ciprian Borcea, Xavier Goaoc, Sylvain Petitjean

\section{To cite this version:}

Ciprian Borcea, Xavier Goaoc, Sylvain Petitjean. Line transversals to disjoint balls. 23rd Annual ACM Symposium on Computational Geometry 2007 - SoCG'07, 2007, Gyeongju, South Korea. pp.245-254, 10.1145/1247069.1247115 . inria-00176201

\section{HAL Id: inria-00176201 https://hal.inria.fr/inria-00176201}

Submitted on 2 Oct 2007

HAL is a multi-disciplinary open access archive for the deposit and dissemination of scientific research documents, whether they are published or not. The documents may come from teaching and research institutions in France or abroad, or from public or private research centers.
L'archive ouverte pluridisciplinaire HAL, est destinée au dépôt et à la diffusion de documents scientifiques de niveau recherche, publiés ou non, émanant des établissements d'enseignement et de recherche français ou étrangers, des laboratoires publics ou privés. 


\section{Line Transversals to Disjoint Balls}

[Extended Abstract]

\author{
Ciprian Borcea \\ Department of Mathematics \\ Rider University \\ Lawrenceville, NJ 08648, USA \\ borcea@rider.edu
}

\author{
Xavier Goaoc \\ LORIA - INRIA Lorraine, \\ Nancy, France \\ Xavier.Goaoc@loria.fr
}

\author{
Sylvain Petitjean \\ LORIA - CNRS, Nancy, France \\ Sylvain.Petitjean@loria.fr
}

\begin{abstract}
We prove that the set of directions of lines intersecting three disjoint balls in $\mathbb{R}^{3}$ in a given order is a strictly convex subset of $\mathbb{S}^{2}$. We then generalize this result to $n$ disjoint balls in $\mathbb{R}^{d}$. As a consequence, we can improve upon several old and new results on line transversals to disjoint balls in arbitrary dimension, such as bounds on the number of connected components and Helly-type theorems.
\end{abstract}

\section{Categories and Subject Descriptors}

F.2.2 [Nonnumerical Algorithms and Problems]: Geometrical problems and computations

\section{General Terms}

Theory

\section{Keywords}

Geometric transversal theory, convexity, lines, disjoint balls, Helly-type theorem, Hadwiger-type theorem, Hessian.

\section{INTRODUCTION}

Helly's theorem [11] of 1923 opened a large field of inquiry now designated as geometric transversal theory. A typical concern is the study of all $k$-planes (also called $k$-flats) which intersect all sets of a given family of subsets (or objects) in $\mathbb{R}^{d}$. These are the $k$-transversals of the given family and they define a certain subspace of the corresponding Grassmannian. True to its origin, transversal theory usually implicates convexity in some form: either in its assumptions, or in its proofs, or, most likely, in both.

In what follows, $k=1$ and the objects will be disjoint closed balls with arbitrary radii in $\mathbb{R}^{d}$. Our main result is the following convexity theorem:

TheOREM 1. The directions of all oriented lines intersecting a given finite family of disjoint balls in $\mathbb{R}^{d}$ in a specific order form a strictly convex subset of the sphere $\mathbb{S}^{d-1}$.

Permission to make digital or hard copies of all or part of this work for personal or classroom use is granted without fee provided that copies are not made or distributed for profit or commercial advantage and that copies bear this notice and the full citation on the first page. To copy otherwise, to republish, to post on servers or to redistribute to lists, requires prior specific permission and/or a fee.

SCG'07, June 6-8, 2007, Gyeongju, South Korea.

Copyright 2007 ACM 978-1-59593-705-6/07/0006 ...\$5.00. with the immediate consequence that the connected components in the space of line transversals correspond with all possible geometric permutations of the given family, where a geometric permutation is understood as a pair of orderings defined by a single line transversal with its two orientations.

Before discussing other implications, we want to emphasize that the key to our theorem resides in the case of three disjoint balls in $\mathbb{R}^{3}$, and the approach we use to settle this case is geometrically quite revealing, in that it shows the nuanced dependency of the convexity property on the curve of common tangents to the three bounding spheres.

\subsection{Relations with previous work}

Helly's theorem [11] states that a finite family $\mathcal{S}$ of convex sets in $\mathbb{R}^{d}$ has non-empty intersection if and only if any subfamily of size at most $d+1$ has non-empty intersection. Passing from $k=0$ to $k=1$, one of the early results is due to Danzer [7] who proved that $n$ disjoint unit disks in the plane have a line transversal if and only if every five of them have a line transversal. Hadwiger's theorem [9], which allows arbitrary disjoint convex sets in the plane as objects, showed the importance of the order in which oriented line transversals meet the objects: when every three objects have an oriented line transversal respecting some fixed order of the whole family, there must be a line transversal for the family.

This stimulated the interest of comparing, in arbitrary dimension, two equivalence relations for line transversals: the coarser one, geometric permutation, determined by the order in which the given disjoint objects are met (up to reversal of orientation), and the finer one, isotopy, determined by the connected components of the space of transversals.

In general, for $d \geqslant 3$, the "gap" between the two notions may be wide [8], and families for which the two notions coincide are thereby "remarkable". The first examples of such families are "thinly distributed" balls ${ }^{1}$ in arbitrary dimension, as observed by Hadwiger [10]. Then, the work of Holmsen et al. [13] showed that disjoint unit balls in $\mathbb{R}^{3}$ provide "remarkable" cases as well. They verified the convexity property in the case of equal radii, and their method can be extended to the larger class of "pairwise inflatable" balls ${ }^{2}$ in arbitrary dimension [5], inviting the obvious question regarding disjoint balls of arbitrary radii. The significance of this

\footnotetext{
${ }^{1} \mathrm{~A}$ family of balls is thinly distributed if the distance between the centers of any two balls is at least twice the sum of their radii.

${ }^{2} \mathrm{~A}$ family of balls is pairwise inflatable if the squared distance between the centers of any two balls is at least twice the sum of their squared radii.
} 
problem is also discussed in the recent notes [18, pg 191-195] where one can find ampler references to related literature.

Our solution for the case of arbitrary radii is based on a new approach, suggested by the detailed study of the curve of common tangents to three spheres in $\mathbb{R}^{3}[2]$. The main ideas are outlined in Section 3 as a preamble to the detailed proof in Section 4.

In dimension three, particularly, there are connections with other problems in visibility and geometric computing. Changes of visibility (or "visual events") in a scene made of smooth obstacles typically occur for multiple tangencies between a line and some of the obstacles [19]. Tritangent and quadritangent lines play a prominent role in this picture, as they determine the 1- and 0-dimensional faces of visibility structures. An attractive case is that of four balls in $\mathbb{R}^{3}$, which allow, generically, up to twelve common real tangents [16]. Degenerate configurations are identified in [3]. Variations on such problems, where reliance on algebraic geometry comes to the forefront, are surveyed in [21]. See also a brief account in [1].

\subsection{Further implications}

Danzer's theorem [7] motivated several other attempts to generalize Helly's result for $k=1$, i.e. for line transversals. Whereas Helly's theorem only requires convexity, the case $k=1$ appears to be more sensitive to the geometry of the objects. In particular, Holmsen and Matoušek [14] showed that no such theorem holds in general for families of disjoint translates of a convex set - not even with restriction on the ordering à la Hadwiger. Our Theorem 1 has consequences in this direction, presented below in Section 5 .

Hadwiger's proof of his Transversal Theorem [9] relies on the observation that any minimal pinning configuration, i.e. family of objects with an isolated line transversal that would become non-isolated should any of the objects be removed, has size 3 if the objects are disjoint convex sets in the plane. Theorem 1 implies that any minimal pinning configuration of disjoint balls in $\mathbb{R}^{d}$ has size at most $2 d-1$ (Corollary 13). A generalization of Hadwiger's theorem for families of disjoint balls then follows (Corollary 14).

\section{PRELIMINARIES}

\subsection{Notations and prerequisites}

For any two vectors $a, b$ of $\mathbb{R}^{3}$, we denote by $\langle a, b\rangle$ their dot product and by $a \times b$ their cross product.

The space of directions in $\mathbb{R}^{3}$ is the real projective space $\mathbb{P}^{2}=\mathbb{P}^{2}(\mathbb{R})$ envisaged either as the space of lines through the origin (and then the direction of a line is given by its parallel through the origin), or as the "plane at infinity" in the completion $\mathbb{P}^{3}=\mathbb{R}^{3} \sqcup \mathbb{P}^{2}$ (and then the direction of a line is simply its point of intersection with the plane at infinity). Convexity in $\mathbb{P}^{2}$ is relative to the metric induced from the standard metric of the sphere through the identification $\mathbb{S}^{2} / \mathbb{Z}_{2}=\mathbb{P}^{2}$. All considerations can be pulled-back to $\mathbb{S}^{2}$ by orienting the lines.

In following our convexity arguments related to three disjoint balls in $\mathbb{R}^{3}$, it may be helpful to bear in mind that the regions of $\mathbb{P}^{2}$ determined by directions of line transversals are always contained in the simply-connected side of some smooth conic (which is homeomorphic with a disc, while the other side is homeomorphic with a Möbius band). When testing convexity, one may use affine charts $\mathbb{R}^{2}$, and verify locally, then globally, that the boundary curve "stays on the same side of its tangent". If this property were to fail at some point, one must have an inflection point there, or, in one word, a flex.

We denote by $B_{0}, B_{1}, B_{2}$ three balls in $\mathbb{R}^{3}$ with respective centers $c_{0}, c_{1}, c_{2}$ and squared radii $s_{0}, s_{1}, s_{2}, s_{k}=r_{k}^{2}$. Since the degenerate case of collinear centers is easily obtained from the generic case, we assume that we have a non-degenerate triangle of centers.

\subsection{Direction-sextic}

The directions of common tangent lines to $B_{0}, B_{1}, B_{2}$ make up an algebraic curve of degree six in $\mathbb{P}^{2}$, which we call the direction-sextic and denote by $\sigma$. To take advantage of symmetries in expressing $\sigma$, we introduce the edge vectors $e_{i j}=c_{j}-c_{i}$, denote by $\delta_{i j}=\left\langle e_{i j}, e_{i j}\right\rangle$ their squared norms and put

$$
\begin{gathered}
q=q(u)=\langle u, u\rangle, \\
t_{i j}=t_{j i}=\left\langle e_{i j} \times u, e_{i j} \times u\right\rangle=\delta_{i j} q-\left\langle e_{i j}, u\right\rangle^{2} .
\end{gathered}
$$

Thus in $\mathbb{P}^{2}(\mathbb{C})$, the equation $t_{i j}=0$ gives the two tangents from $e_{i j}$ to the imaginary conic $q=0$.

Proposition 2. The direction-sextic for $B_{0}, B_{1}, B_{2}$ can be given by means of the Cayley determinant:

$$
\sigma=\sigma(u)=\operatorname{det}\left(\begin{array}{ccccc}
0 & 1 & 1 & 1 & 1 \\
1 & 0 & q s_{0} & q s_{1} & q s_{2} \\
1 & q s_{0} & 0 & t_{01} & t_{02} \\
1 & q s_{1} & t_{01} & 0 & t_{12} \\
1 & q s_{2} & t_{02} & t_{12} & 0
\end{array}\right)=0
$$

Proof. One way to find the equation of the direction curve is to begin with a description of lines in $\mathbb{R}^{3}$ by parameters $(p, u) \in \mathbb{R}^{3} \times \mathbb{P}^{2}$, where $p$ is the orthogonal projection of the origin on the given line, and $u$ is the direction of the line. With $c_{0}=0$ and abbreviations:

$$
\begin{aligned}
a_{i}=a_{i}(u) & =\left\langle c_{i} \times u, c_{i} \times u\right\rangle+\left(s_{0}-s_{i}\right)\langle u, u\rangle, \\
& =t_{0 i}+\left(s_{0}-s_{i}\right) q, \quad i=1,2,
\end{aligned}
$$

affine common tangents obey the system (see e.g. [3] or $[16])$ :

$$
\left\langle p, c_{i}\right\rangle=\frac{a_{i}(u)}{2\langle u, u\rangle}, \quad i=1,2, \quad\langle p, u\rangle=0, \quad\langle p, p\rangle=s_{0} .
$$

The direction-sextic is obtained by eliminating $p$ from this system. The fact that the resulting equation allows the stated Cayley determinant expression is given a natural explanation in [2], but can be directly verified by computation.

The direction of an oriented line can be represented either by a point on the unit sphere, or by the whole ray emanating from the origin and passing through that point. Our expression "cone of directions" stems from the latter representation, which converts questions of convexity in $\mathbb{S}^{2}$ into equivalent questions of convexity in $\mathbb{R}^{3}$. In the projective context, it will be understood that we mean the image via $\mathbb{S}^{2} / \mathbb{Z}_{2}=\mathbb{P}^{2}$.

\subsection{Cone of directions}

The cone of directions $K\left(B_{0} B_{1} B_{2}\right)$ of $B_{0}, B_{1}, B_{2}$ is the set of directions of all oriented line transversals to these balls 


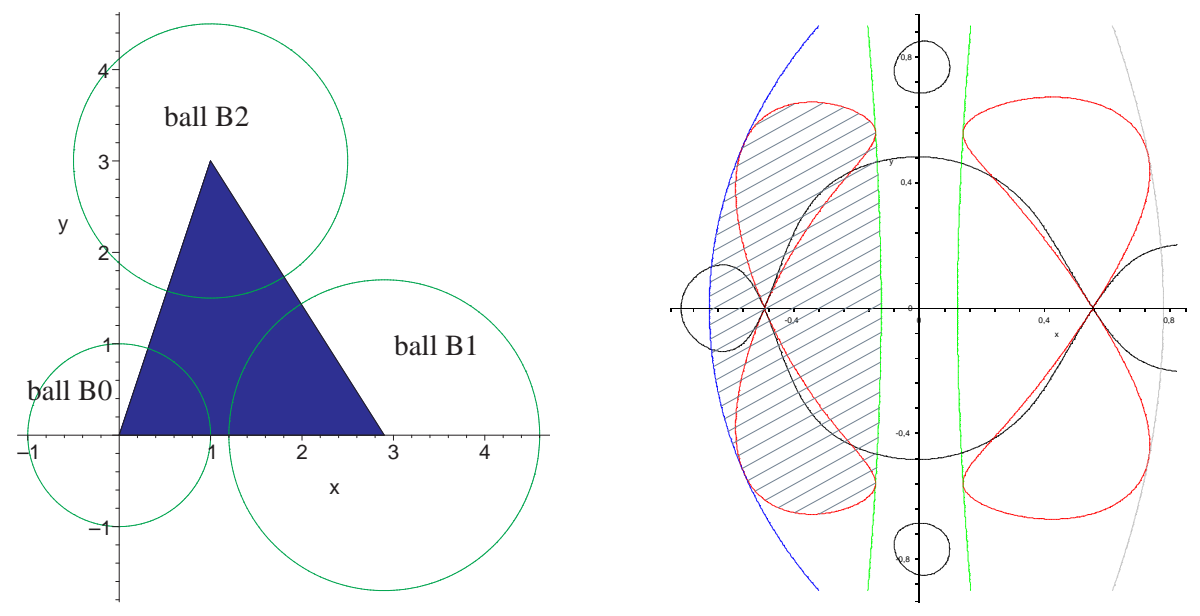

Figure 1: A configuration of three balls (left) and a planar depiction of a cone of directions (right). The direction-sextic is in red, and the Hessian in black. The hatched region corresponds to directions of transversals to the three balls in the order $B_{1} \prec B_{0} \prec B_{2}$, the blue arc corresponding to inner special bitangents of balls $B_{0}$ and $B_{2}$ and the green arc corresponding to inner special bitangents of balls $B_{0}$ and $B_{1}$.

which meet them in the stated order: $B_{0} \prec B_{1} \prec B_{2}$. The boundary of $K\left(B_{0} B_{1} B_{2}\right)$ consists of [5, Lemma 9] certain arcs of the direction-sextic $\sigma$, and certain arcs of directions of inner special bitangents i.e. tangents to two of the balls passing through their inner similitude center [12]. Figure 1 offers an illustration of a cone of directions. The plane of the picture must be conceived as an affine piece $\mathbb{R}^{2} \subset \mathbb{P}^{2}$.

We recall the fact that a common tangent (here called bitangent) for two disjoint spheres (more precisely, the boundary of two disjoint balls) passes through their inner similitude center if and only if it is contained in a common tangent plane which has the two spheres on opposite sides. If a transversal for the two balls has the direction of an inner special bitangent, it must actually be that bitangent. The cone of directions for a pair of disjoint balls is bounded precisely by their inner special bitangents. In $\mathbb{P}^{2}$ they trace a (circular) conic.

The points of $\sigma$ that appear on the boundary $\partial K\left(B_{0} B_{1} B_{2}\right)$ can be characterized as follows:

Proposition 3. The direction of a tritangent $\ell$ meeting the three balls $B_{0}, B_{1}, B_{2}$ in the prescribed order belongs to $\partial K\left(B_{0} B_{1} B_{2}\right)$ if and only if $\ell$ intersects the triangle of centers $c_{0} c_{1} c_{2}$.

Proof. The set of directions of common transversals to disjoint balls is a proper subset of $\mathbb{P}^{2}$.

Assume that $\ell$ is neither parallel to the plane of centers, nor contained in it.

If the intercept point of $\ell$ with the plane of centers lies outside the triangle of centers, there exists an edge which has a center on the other side. This still holds for the projected configuration on $\ell^{\perp}$. When moving along the perpendicular closer to the projected edge, all distances to projected centers decrease. This shows that there are lines parallel to $\ell$ "stabbing" the open balls, and therefore the direction of $\ell$ is not on the boundary. On the other hand, when the tritangent $\ell$ intersects the triangle of centers, and we follow the projection along $\ell$ on $\ell^{\perp}$, there is no distance decreasing motion for all distances to the (projected) vertices, for this would decrease all areas over edges, while these areas have a constant sum. Thus the direction of $\ell$ is on $\partial K\left(B_{0} B_{1} B_{2}\right)$.
In other words, the general case follows from the elementary statement that given a (top dimensional) simplex in a Euclidean space, and a point, the balls centered at the vertices of the simplex and passing through the given point intersect only at that point when it belongs to the simplex, but have an intersection with non-empty interior when the point is outside the simplex.

If $\ell$ is parallel to the plane of centers (but not contained in it), we may consider any parallel plane which is closer to $c_{0} c_{1} c_{2}$ than $\ell$ is, and find in this plane transversals to the open balls parallel to $\ell$. Thus, $\ell$ cannot be on the boundary.

Finally, if $\ell$ is in the plane of centers, and we look at the "section configuration" traced in that plane, either all three discs are on one side of $\ell$ and then $\ell$ does not cross the triangle of centers and is not on the boundary, or $\ell$ has two discs on one side and the third on the other, must cross the triangle of centers, is actually an inner special bitangent for two pairs of balls (and an outer special bitangent for the third pair), and is thus necessarily on the boundary.

Proposition 4. For three disjoint balls, we have:

(i) the cone of directions $K\left(B_{0} B_{1} B_{2}\right)$ consists of a single point if and only if there is a tritangent contained in the plane of centers, and tracing in it a pinned planar configuration i.e. the disc traced by $B_{1}$ is on the other side of the tritangent than the discs traced by $B_{0}$ and $B_{2}$;

(ii) in all other cases, the cone of directions $K\left(B_{0} B_{1} B_{2}\right)$ is the closure of its interior.

Proof. (i) Sufficiency: the plane intersecting the plane of centers along the tritangent, and perpendicular to it, will have $B_{1}$ on one side, and $B_{0}$ and $B_{2}$ on the other. An oriented transversal meeting $B_{0}$ first, then $B_{1}$, and then $B_{2}$ must be contained in this separating perpendicular plane, and thus coincide with the given tritangent. Necessity is covered by our arguments in (ii).

(ii) Suppose we are not in case (i), and the centers are not aligned. If we have a transversal $\ell$ with direction belonging to the boundary of $K\left(B_{0} B_{1} B_{2}\right)$, we may assume the 
transversal is not in the plane of centers, since a non-pinned planar case is clear. But then $\ell$ and its reflection in the plane of centers define a plane perpendicular to the latter, and all lines between them (passing through their intersection) have directions belonging to the interior, because all distances from centers decrease.

The case of collinear centers is trivial: there is only one geometric permutation (given by the line of centers) and the cone of directions is a disc-like region bounded by a conic.

Corollary of Proof. Cone of directions and connected components of transversals for three disjoint balls in $\mathbb{R}^{3}$ are contractible.

Indeed, the argument above shows that we may contract first to the segment in $K\left(B_{0} B_{1} B_{2}\right)$ consisting of directions in the plane of centers, and then contract this segment.

Obviously the same holds true at the level of the connected components in the space of transversals.

\subsection{Hessian and flexes}

The Hessian of $\sigma$ is defined as the determinant of the matrix of second derivatives:

$$
H(\sigma)=H(\sigma)(u)=\operatorname{det}\left(\frac{\partial^{2} \sigma}{\partial u_{i} \partial u_{j}}\right) .
$$

The Hessian curve (or simply Hessian) is the projective curve defined by the zero-set of this determinant.

The Hessian of a direction-sextic for three balls in $\mathbb{R}^{3}$ is thus an algebraic curve of degree twelve. The intersection between $\sigma$ and its Hessian $H(\sigma)$ consists of all singular points of $\sigma$ and all flexes of $\sigma$ [4].

\section{OUTLINE OF THE PROOF}

For $d=2$ the convexity theorem is elementary, and for $d \geqslant 3$ it is easily reduced to the case of three disjoint balls in $\mathbb{R}^{3}$. The key property used to settle this case is the following:

Proposition 5. For disjoint balls $B_{0}, B_{1}, B_{2}$, any arc of their direction-sextic $\sigma$ which belongs to the boundary $\partial K\left(B_{0} B_{1} B_{2}\right)$ contains no flex or singularity of $\sigma$ between its endpoints.

The convexity of the cone of directions $K\left(B_{0} B_{1} B_{2}\right)$ can then be inferred from the known fact that a simple $C^{1}$-loop in $\mathbb{R}^{2} \subset \mathbb{P}^{2}$ with no inflection (in Euclidean terms: with positive curvature on its algebraic arcs) bounds a convex interior [22].

Thus, what is essential for this approach, is to obtain sufficient control over the flexes of $\sigma$. At first sight, the fact that the intersection of $\sigma$ and the Hessian $H(\sigma)$ in $\mathbb{P}^{2}(\mathbb{C})$ has (counting multiplicities) $6 \times 12=72$ points leaves little hope for the possibility of "tracking" all flexes. However, there is another way to exploit the Hessian: fix a direction and consider the ball configurations which have a tritangent with that direction and give the same planar configuration of four points when projecting, tangent and centers, on some orthogonal plane; express the Hessians of the corresponding direction-sextics and then ask which may vanish for the given direction.

The important point is that one can anticipate, from the form of the equations, that the computations must result in polynomials of low degree, which will be subject, in their turn, to geometrical control.
The unfolding of this scenario is presented in the next section and involves a certain amount of explicit computations. Although no part is too complicated to be done by hand, we have relied on Maple [17] in a few instances, which are documented in the Appendix.

\section{DETAILS OF THE PROOF}

\subsection{Probing for flexes}

Following Proposition 3, we need only consider directions of tangents to the three balls that cross the triangle of centers and are not directions of inner special bitangents. When projecting along such a tangent on a perpendicular plane, the projected centers form a triangle containing the point image of the tangent as an interior point. One may start with the latter planar configuration, a triangle and an interior point, and ask: what ball configurations yield this picture (by projection along a common tangent intersecting at the interior point)? Since the radii of the balls are given, one has only to "lift" the vertices of the triangle in the normal direction and obtain all the asked for configurations.

We equip $\mathbb{R}^{3}$ with a frame such that the triangle lies in the plane $e_{3}^{\perp} \subset \mathbb{R}^{3}$ and has its vertices at $\tilde{c}_{0}=0, \tilde{c}_{1}, \tilde{c}_{2}$, with the understanding that there is a point inside, with squared distances $s_{i}$ to these vertices. Then, we use three real parameters, $x_{0}, x_{1}$ and $x_{2}$, to describe the possible positions of the three centers:

$$
c_{0}=\tilde{c}_{0}+x_{0} e_{3}, \quad c_{1}=\tilde{c}_{1}+x_{1} e_{3}, \quad c_{2}=\tilde{c}_{2}+x_{2} e_{3} .
$$

We use Proposition 2 to express the corresponding direction sextic $\sigma$ and its Hessian $H(\sigma)$ as functions of $x=\left(x_{0}, x_{1}, x_{2}\right) \in \mathbb{R}^{3}$ depending on $\tilde{c}_{0}, \tilde{c}_{1}, \tilde{c}_{2}, s_{0}, s_{1}, s_{2}$. Proposition 5 is now equivalent to proving that

$$
H(\sigma)(0,0,1) \neq 0
$$

holds for all initial data (triangle and interior point) and all $\left(x_{0}, x_{1}, x_{2}\right)$ corresponding to disjoint balls.

\subsection{A quadric and a quartic}

We have reduced the probe for flexes to the study of a polynomial function of $x$ (and parameters) which can be explicitly computed. For the Maple procedure we used, see Appendix.

The parameters involved are the following:

$$
\tilde{c}_{0}=(0,0,0), \quad \tilde{c}_{1}=(a, 0,0), \quad \tilde{c}_{2}=(b, c, 0),
$$

the triangle of centers $\left(\tilde{c}_{0}, \tilde{c}_{1}, \tilde{c}_{2}\right)$ having interior point:

$$
p=\frac{\sum p_{i} \tilde{c}_{i}}{\sum p_{i}}=\frac{p_{1} \tilde{c}_{1}+p_{2} \tilde{c}_{2}}{\sum p_{i}}, \quad p_{0}, p_{1}, p_{2}>0 .
$$

Let $v_{k}=p-\tilde{c}_{k}$. Then $s_{k}=r_{k}^{2}=\left\langle v_{k}, v_{k}\right\rangle$.

The computation gives the result:

$$
H(\sigma)(0,0,1)=\frac{2^{12} 5^{2} a^{6} c^{6}}{\left(\sum p_{i}\right)^{5}}\left[H_{2}(x)+H_{4}(x)\right],
$$

where $H_{2}$ and $H_{4}$ have degree respectively 2 and 4 in $x=\left(x_{0}, x_{1}, x_{2}\right)$ :

$$
\begin{gathered}
H_{2}=H_{2}(x)=-a^{2} c^{2} \prod p_{k} \sum p_{i} p_{j}\left(x_{i}-x_{j}\right)^{2}, \\
H_{4}=H_{4}(x)=\sum p_{k}^{3} s_{k}\left(x_{i}-x_{k}\right)^{2}\left(x_{j}-x_{k}\right)^{2},
\end{gathered}
$$


with cyclic notation for $\{i, j, k\}=\{0,1,2\}$. Thus, away from $(0,0,0), H_{2}$ is negative and $H_{4}$ is positive. The aim is now to show that the assumption of disjoint balls is enough to ensure the positivity of $\mathrm{H}_{2}+\mathrm{H}_{4}$.

\subsection{Hyperboloid and octant}

We can further transform these expressions by retaining as parameters the (positive numbers) $p_{i}$ and $q_{j}=p_{j} r_{j}$, and renaming the squares $z_{k}=\left(x_{i}-x_{j}\right)^{2}$. This gives:

$$
\begin{gathered}
H_{2}=H_{2}(z)=-a^{2} c^{2} \prod p_{k} \sum p_{i} p_{j} z_{k}, \\
H_{4}=H_{4}(z)=\sum p_{k} q_{k}^{2} z_{i} z_{j} .
\end{gathered}
$$

From now on, assume that $\sum p_{i}=1$. We have to replace $\Delta=a^{2} c^{2}$, which is four times the squared area of the triangle $\tilde{c}_{0}, \tilde{c}_{1}, \tilde{c}_{2}$, by its expression in terms of $p_{i}$ and $q_{j}$.

LEMMA 6. We have:

$$
\Delta=a^{2} c^{2}=\frac{Q}{4 \prod p_{k}^{2}}, \quad \text { with } \quad Q=\sum\left(2 q_{i}^{2} q_{j}^{2}-q_{k}^{4}\right) .
$$

Proof. This is an elementary computation, which may be conducted as follows. By the definition of $v_{i}$, we have

$$
\sum p_{i} v_{i}=0
$$

From $\left\langle\sum p_{i} v_{i}, v_{j}\right\rangle=0$, we obtain a linear system for $\left\langle v_{i}, v_{j}\right\rangle, i \neq j$ :

$$
p_{i}\left\langle v_{i}, v_{k}\right\rangle+p_{j}\left\langle v_{j}, v_{k}\right\rangle=-p_{k}\left\langle v_{k}, v_{k}\right\rangle=-p_{k} s_{k},
$$

with solutions:

$$
\left\langle v_{i}, v_{j}\right\rangle=\frac{p_{k}^{2} s_{k}-p_{i}^{2} s_{i}-p_{j}^{2} s_{j}}{2 p_{i} p_{j}}=\frac{q_{k}^{2}-q_{i}^{2}-q_{j}^{2}}{2 p_{i} p_{j}} .
$$

Four times the squared area of a triangle $p, \tilde{c}_{i}, \tilde{c}_{j}$ is a Gram determinant:

$$
\left|\begin{array}{cc}
\left\langle v_{i}, v_{i}\right\rangle & \left\langle v_{i}, v_{j}\right\rangle \\
\left\langle v_{i}, v_{j}\right\rangle & \left\langle v_{j}, v_{j}\right\rangle
\end{array}\right|=s_{i} s_{j}-\left\langle v_{i}, v_{j}\right\rangle^{2}=\frac{Q}{4 p_{i}^{2} p_{j}^{2}},
$$

where $Q=\sum\left(2 q_{i}^{2} q_{j}^{2}-q_{k}^{4}\right)$. Hence the area of the triangle $\tilde{c}_{0}, \tilde{c}_{1}, \tilde{c}_{2}$ is:

$$
\frac{1}{4} Q^{1 / 2} \sum \frac{1}{p_{i} p_{j}}=\frac{Q^{1 / 2}}{4 \prod p_{k}}
$$

resulting in:

$$
\Delta=a^{2} c^{2}=\frac{Q}{4 \prod p_{k}^{2}}
$$

Several new substitutions will be in order for the study of $H_{2}+H_{4}$. Since a positive factor won't affect sign considerations, we'll use the symbol $* H$ for any positive multiple of $H_{2}+H_{4}$. We have found above:

$$
* H=* H(z)=-\frac{1}{4} Q \sum \frac{z_{k}}{p_{k}}+\sum p_{k} q_{k}^{2} z_{i} z_{j},
$$

with the shorthand $Q=\sum\left(2 q_{i}^{2} q_{j}^{2}-q_{k}^{4}\right)$. We put $p_{i} p_{j} z_{k}=q_{k}^{2} w_{k}$, and obtain (up to a positive factor):

$$
* H=* H(w)=-\frac{1}{4} Q \sum q_{k}^{2} w_{k}+\prod q_{k}^{2} \sum w_{i} w_{j} .
$$

With one more positive rescaling, and $a_{k}=\frac{Q}{4 q_{i}^{2} q_{j}^{2}}$, we have:

$$
* H=* H(w)=\sum w_{i} w_{j}-\sum a_{k} w_{k} .
$$

We can turn now to the conditions expressing the fact that the spheres with centers $c_{i}=\tilde{c}_{i}+x_{i} e_{3}$ and radii $r_{i}$ are disjoint. They are:

$z_{k}=\left(x_{i}-x_{j}\right)^{2}>\left(r_{i}+r_{j}\right)^{2}-\delta_{i j}=\left(r_{i}+r_{j}\right)^{2}-\left\langle v_{i}-v_{j}, v_{i}-v_{j}\right\rangle$,

that is:

$$
z_{k}>\frac{q_{k}^{2}-\left(q_{i}-q_{j}\right)^{2}}{p_{i} p_{j}} .
$$

In $w$-coordinates, the "disjointness conditions" become

$$
w_{k}>1-\left(\frac{q_{i}-q_{j}}{q_{k}}\right)^{2} .
$$

Note that from $\sum p_{i} v_{i}=0$ it follows that $q_{k}=\left\|p_{i} v_{i}\right\|>0$ are the edges of a triangle, and therefore the latter expressions are positive by the triangle inequality.

The purpose now is to study the position of the octant defined by the "disjointness conditions" relative to the affine quadric in $\mathbb{R}^{3}$ defined by $* H(w)=0$. We use first a translation by $\beta$, in order to absorb the linear part in $* H$ :

$$
* H=* H(w)=\sum\left(w_{i}-\beta_{i}\right)\left(w_{j}-\beta_{j}\right)-\sum \beta_{i} \beta_{j},
$$

requesting:

$$
\beta_{i}+\beta_{j}=a_{k}, \text { that is } \beta_{k}=\frac{1}{2}\left(a_{i}+a_{j}-a_{k}\right) .
$$

This makes

$$
\begin{aligned}
\sum \beta_{i} \beta_{j} & =\frac{1}{4} \sum\left(a_{k}+a_{i}-a_{j}\right)\left(a_{k}-a_{i}+a_{j}\right), \\
& =\frac{1}{4} \sum\left(2 a_{i} a_{j}-a_{k}^{2}\right),
\end{aligned}
$$

and results in

$$
\sum \beta_{i} \beta_{j}=\frac{1}{4}\left(\frac{Q}{4 \prod q_{k}^{2}}\right)^{2} \sum\left(2 q_{i}^{2} q_{j}^{2}-q_{k}^{4}\right)=\frac{Q^{3}}{4^{3} \prod q_{k}^{4}}>0 .
$$

Thus, with translated coordinates $t_{k}=w_{k}-\beta_{k}$ we have a hyperboloid with two sheets:

$$
* H=* H(t)=\sum t_{i} t_{j}-\frac{Q^{3}}{4^{3} \prod q_{k}^{4}}=0,
$$

which lies on the positive side of its asymptotic cone $\sum t_{i} t_{j}=0$.

LEMMA 7. $\sum t_{i} t_{j}=0$ is a circular cone with axis $t_{0}=t_{1}=t_{2}$. The two components of its smooth points circumscribe the positive and negative open octants, which are both contained in the positive part $\sum t_{i} t_{j}>0$.

The open octant defined by our "disjointness conditions" $w_{k}>1-\left(\frac{q_{i}-q_{j}}{q_{k}}\right)^{2}$ is a translate of the open positive octant, and its position relative to the hyperboloid $* H(w)=0$ is determined by the position of its vertex $V$. Continuing to refer here to $w$-coordinates, we have:

Lemma 8. The point $V=\left(1-\left(\frac{q_{i}-q_{j}}{q_{k}}\right)^{2}\right)_{0 \leqslant k \leqslant 2}$ is on the "positive side" of the hyperboloid $* H(w)=0$ and on the 
"positive side" of the plane $\sum t_{k}=\sum\left(w_{k}-\beta_{k}\right)=0$, that is:

$* H(V)>0 \quad$ and $\quad \sum\left(1-\left(\frac{q_{i}-q_{j}}{q_{k}}\right)^{2}\right)>\frac{Q}{8 \prod q_{k}^{2}} \sum q_{k}^{2}$.

Proof. A Maple assisted computation (see Appendix) shows that $* H(V)$ factors as

$$
* H(V)=\frac{3 \prod\left(q_{i}+q_{j}-q_{k}\right)^{2}}{4 \prod q_{k}^{2}},
$$

from which the first inequality follows.

The second inequality, which determines on which of the two components of the "positive side" of the hyperboloid $V$ lies, is satisfied for $q_{0}=q_{1}=q_{2}$, and by continuity, must be satisfied for any other triangle edges, since vertex $V$ cannot "jump" from one component to the other.

It is now clear, geometrically, that the octant where the "disjointness conditions" are satisfied and the hyperboloid indicating a flex or a singularity for the corresponding configuration have no point in common. This completes the proof of Proposition 5.

\subsection{Convexity of the cone of directions}

We consider now three disjoint closed balls $B_{0}, B_{1}, B_{2}$ described by parameters: centers $c_{0}, c_{1}, c_{2}$ and radii $r_{0}, r_{1}, r_{2}$. We shall prove first the convexity of any cone of directions in the generic case i.e. when the centers and radii are in the complement of a proper algebraic subset. Then, we will show that the generic case implies the general case.

Lemma 9. The direction cone $K\left(B_{0} B_{1} B_{2}\right)$ of a generic triple of disjoint balls in $\mathbb{R}^{3}$ is strictly convex.

Proof. If $\partial K\left(B_{0} B_{1} B_{2}\right)$ is made only of directions of inner special bitangents, strict convexity is immediate, since $K\left(B_{0} B_{1} B_{2}\right)$ is then an intersecion of convex regions bounded by conics. Otherwise, genericity allows us to assume that the direction sextic $\sigma$ is non-singular at all its contacts with any of the three conics determined by inner special tangents. Then, these contacts are tangency points, and if we start at some point of, say $\partial K\left(B_{0} B_{1} B_{2}\right)$, and follow the boundary curve, we obtain, by Proposition 5 , a differentiable simple loop of class $C^{1}$, which is, locally, always on the same side of its tangent. For any affine plane $\mathbb{R}^{2} \subset \mathbb{P}^{2}$ covering the loop, and any Euclidean metric in it, this means positive curvature on all its algebraic arcs, and this implies [22] the fact that our simple loop bounds a compact convex set. In fact strictly convex, because of nonvanishing curvature. By Proposition 4 and its Corollary, this strictly convex set is $K\left(B_{0} B_{1} B_{2}\right)$.

The passage from the generic case to the general case is based on:

Lemma 10 . Let $\mathcal{B}=\left(B_{0}, B_{1}, B_{2}\right)$ be a configuration of three disjoint closed balls, and suppose $K\left(B_{0} B_{1} B_{2}\right)$ has nonempty interior. If $\mathcal{B}$ is the limit of a sequence of configurations $\mathcal{B}^{(\nu)}$ with a convex corresponding cone of directions, then $K\left(B_{0} B_{1} B_{2}\right)$ is convex as well.

Proof. By Proposition 4, it is enough to prove that, for any two points in the interior, the (geodesic) segment joining them is contained in $K\left(B_{0} B_{1} B_{2}\right)$.
Take two interior points. By assumption, for sufficiently large $\nu$, the segment joining them is contained in all corresponding cones for $\mathcal{B}^{(\nu)}$. Consider one point of the segment, and project the sphere configuration along the direction defined by the point, on a perpendicular plane. We have to prove that the disks representing the projected balls have at least one point in common.

Suppose they don't. Then so would discs with the same centers and radii increased by a small $\epsilon>0$. But then we can find, for sufficiently large $\nu$, configurations $\mathcal{B}^{(\nu)}$ with centers projecting less than $\epsilon / 2$ away from those of $\mathcal{B}$, and corresponding radii with less than $\epsilon / 2$ augmentation. Then the point of the segment cannot be in the respective cones of directions: a contradiction.

The convexity result generalizes to arbitrary $n$ and $d$ as follows:

Proof of TheOREM 1. Recall that, for any collection of balls in $\mathbb{R}^{3}$, a direction will be realized by some transversal if and only if the orthogonal projection of the balls on a perpendicular plane has non-empty intersection. By Helly's Theorem in the plane, the direction cone for a sequence of $n \geqslant 3$ balls is the intersection of the direction cones of all its triples. Thus, the direction cone of $n$ ordered 3 -dimensional disjoint balls is strictly convex for any $n$.

Given a sequence $\mathcal{S}$ of $n$ disjoint balls in $\mathbb{R}^{d}$, let $K$ be its direction cone for a prescribed order of intersection. Let $u$ and $v$ be two directions in $K, \ell_{u}$ and $\ell_{v}$ be two corresponding line transversals and let $E$ denote the 3-dimensional affine space these two lines span (or a 3-space containing their planar span, should the lines be coplanar).

$E \cap \mathcal{S}$ is a collection of 3-dimensional disjoint balls whose corresponding direction cone is convex on $\mathbb{S}^{2}$. Thus, for any direction on the small arc of great circle joining $u$ and $v$ there exists an order-respecting transversal to $\mathcal{S}$, because it already exists in $E$. It follows that $K$ is convex, and again, from the three dimensional case, strictly convex.

Let us emphasize the importance of the assumption that the balls are disjoint. Figure 2 illustrates a transition from convex to non-convex direction cones as three disjoint balls move and allow an overlap.

\section{IMPLICATIONS}

This section explores some consequences of Theorem 1 . Similar results were proven for the case of unit balls in [5] and, with Theorem 1, the proofs carry through. We thus omit all arguments here and point to the relevant lemmata in $[5]$.

\subsection{Isotopy and geometric permutations}

An immediate corollary of Theorem 1 is the correspondence of isotopy and geometric permutations for line transversals to disjoint balls:

COROLlary 11. The set of line transversals to $n$ disjoint balls in $\mathbb{R}^{d}$ realizing the same geometric permutation is contractible.

The proof given by Cheong et al. [5, Lemma 14] for disjoint unit balls immediately extends, with Theorem 1 , to the case of disjoint balls. Smorodinsky et al. [20] showed that in the worst case $n$ disjoint balls in $\mathbb{R}^{d}$ admit $\Theta\left(n^{d-1}\right)$ geometric 


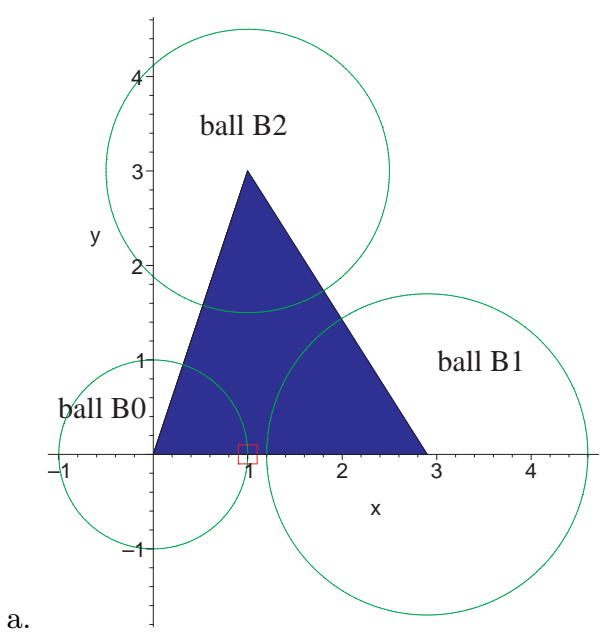

b.


d.
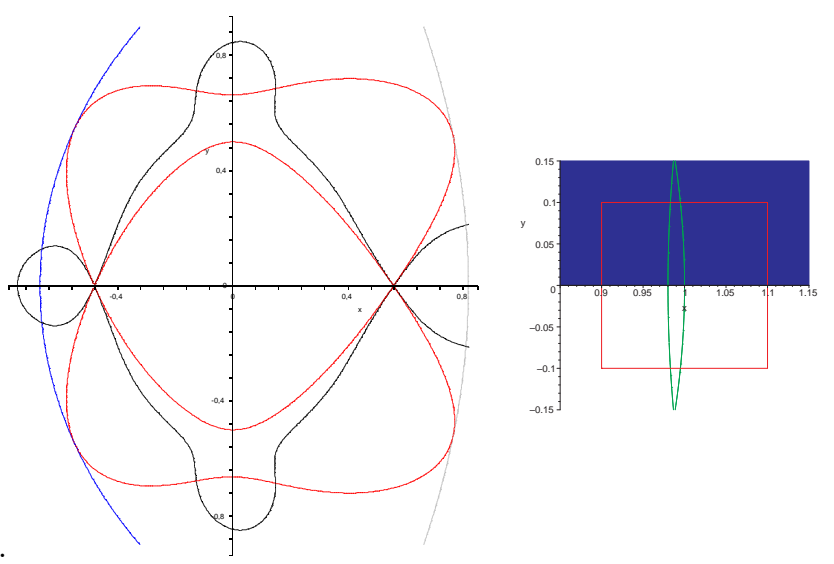

Figure 2: a. The trace of three disjoint balls on the plane of centers, with ball $B_{1}$ moving on the horizontal axis towards ball $B_{0}$. The red square is used for close-ups below. b. c. d. The direction-sextic (in red), its Hessian (in black) and the inner special bitangent conics (in blue, green and gray), when balls $B_{0}$ and $B_{1}$ are disjoint (b), tangent (c) and intersecting (d). 
permutations. The same bound thus applies for the number of connected components of line transversals, improving on the previous bounds of $O\left(n^{3+\epsilon}\right)$ for $d=3$ and of $O\left(n^{2 d-2}\right)$ for $d \geqslant 4$ due to Koltun and Sharir [15]. If the radii of the balls are in some interval $[1, \gamma]$ where $\gamma$ is independent of $n$ and $d$, then the number of components of transversals is $O\left(\gamma^{\log \gamma}\right)$, following the bound on the number of geometric permutations obtained by Zhou and Suri [23]. These results are summarized as follows:

COROLlary 12 . In the worst case, $n$ disjoint balls in $\mathbb{R}^{d}$ have $\Theta\left(n^{d-1}\right)$ connected components of line transversals. If the radii of the balls are in the interval $[1, \gamma]$, where $\gamma$ is independent of $n$ and $d$, this number becomes $O\left(\gamma^{\log \gamma}\right)$.

\subsection{Minimal pinning configurations}

A minimal pinning configuration is a collection of objects having an isolated line transversal that ceases to be isolated if any of the objects is discarded. An important step in the proof of Hadwiger's transversal theorem [9] is the observation that, in the plane, any minimal pinning configuration consisting of disjoint convex objects has cardinality 3. Cheong et al. [5, Proposition 13] proved that any minimal pinning configuration consisting of disjoint unit balls in $\mathbb{R}^{d}$ has cardinality at most $2 d-1$. With Theorem 1 , the same holds for disjoint balls of arbitrary radii:

Corollary 13. Any minimal pinning configuration consisting of disjoint balls in $\mathbb{R}^{d}$ has cardinality at most $2 d-1$.

\subsection{A Hadwiger-type result}

The "pure" generalizations $[5,13]$ of Helly's theorem, i.e. without additional constraints on the ordering à la Hadwiger, use two ingredients: the convexity of the cone of directions and the fact that $n \geqslant 9$ disjoint unit balls have at most 2 geometric permutations [6]. Since the latter is not true for balls of arbitrary radii [20], such theorems do not generalize immediately to non-unit balls. Yet, an intermediate result of independent interest in the flavor of Hadwiger's transversal theorem does generalize:

COROLlary 14. A sequence of $n$ disjoint balls in $\mathbb{R}^{d}$ has a line transversal if any subsequence of size at most $2 d$ has an order-respecting line transversal.

\section{REFERENCES}

[1] C. Borcea. Algebraic geometry for constraint problems. In S. il Pae and H. Park, editors, Proc. 7th Asian Symposium in Computer Math., pages 112-114. 2005.

[2] C. Borcea. Involutive sextics and tangents to spheres. Manuscript, 2006.

[3] C. Borcea, X. Goaoc, S. Lazard, and S. Petitjean. Common tangents to spheres in $\mathbb{R}^{3}$. Discrete and Computational Geometry, 35(2):287-300, 2006.

[4] E. Brieskorn and H. Knörrer. Plane Algebraic Curves. Birkhaüser Verlag, Basel, 1986.

[5] O. Cheong, X. Goaoc, A. Holmsen, and S. Petitjean. Hadwiger and Helly-type theorems for disjoint unit spheres. Discrete and Computational Geometry, 2006. To appear, special issue for the 20th anniversary of the journal.
[6] O. Cheong, X. Goaoc, and H.-S. Na. Geometric permutations of disjoint unit spheres. Comput. Geom. Theory Appl., 30:253-270, 2005.

[7] L. Danzer. Über ein Problem aus der kombinatorischen Geometrie. Arch. der Math, 1957.

[8] J. E. Goodman, R. Pollack, and R. Wenger. Geometric transversal theory. In J. Pach, editor, New Trends in Discrete and Computational Geometry, volume 10 of Algorithms and Combinatorics, pages 163-198. Springer-Verlag, Heidelberg, Germany, 1993.

[9] H. Hadwiger. Über Eibereiche mit gemeinsamer Treffgeraden. Portugal Math., 6:23-29, 1957.

[10] H. Hadwiger. Problem 107. Nieuw Arch. Wisk., (3)4:57, 1956; Solution. Wiskundige Opgaven, 20:27-29, 1957.

[11] E. Helly. Über Mengen konvexer Körper mit gemeinschaftlichen Punkten. Jahresbericht Deutsch. Math. Verein., 32:175-176, 1923.

[12] D. Hilbert and S. Cohn-Vossen. Geometry and the Imagination. Chelsea Pub Co, 1952.

[13] A. Holmsen, M. Katchalski, and T. Lewis. A Helly-type theorem for line transversals to disjoint unit balls. Discrete Comput. Geom., 29:595-602, 2003.

[14] A. Holmsen and J. Matoušek. No Helly theorem for stabbing translates by lines in $\mathbb{R}^{d}$. Discrete Comput. Geom., 31:405-410, 2004.

[15] V. Koltun and M. Sharir. The partition technique for overlays of enveloppes. SIAM Journal of Computing, 32:841-863, 2003

[16] I. Macdonald, J. Pach, and T. Theobald. Common tangents to four unit balls in $\mathbb{R}^{3}$. Discrete Comput. Geom., 26:1-17, 2001.

[17] The Maple System. Waterloo Maple Software. http://www .maplesoft.com.

[18] J. Pach and M. Sharir. Combinatorial Geometry with Algorithmic Applications - The Alcala Lectures. Alcala (Spain), August 31 - September 5, 2006.

[19] O. Platonova. Singularities of the mutual disposition of a surface and a line. Russian Mathematical Surveys, 36:248-249, 1981

[20] S. Smorodinsky, J. S. B. Mitchell, and M. Sharir. Sharp bounds on geometric permutations for pairwise disjoint balls in $\mathbb{R}^{d}$. Discrete Comput. Geom., 23:247-259, 2000.

[21] F. Sottile and T. Theobald. Line problems in nonlinear computational geometry. arXiv math.MG/0610407, 2006.

[22] V. Toponogov. Differential Geometry of Curves and Surfaces: A Concise Guide. Birkhäuser, 2006.

[23] Y. Zhou and S. Suri. Geometric permutations of balls with bounded size disparity. Comput. Geom. Theory Appl., 26:3-20, 2003. 


\section{Appendix: Maple code}

Maple 10 code [17] for computations used in Section 4.

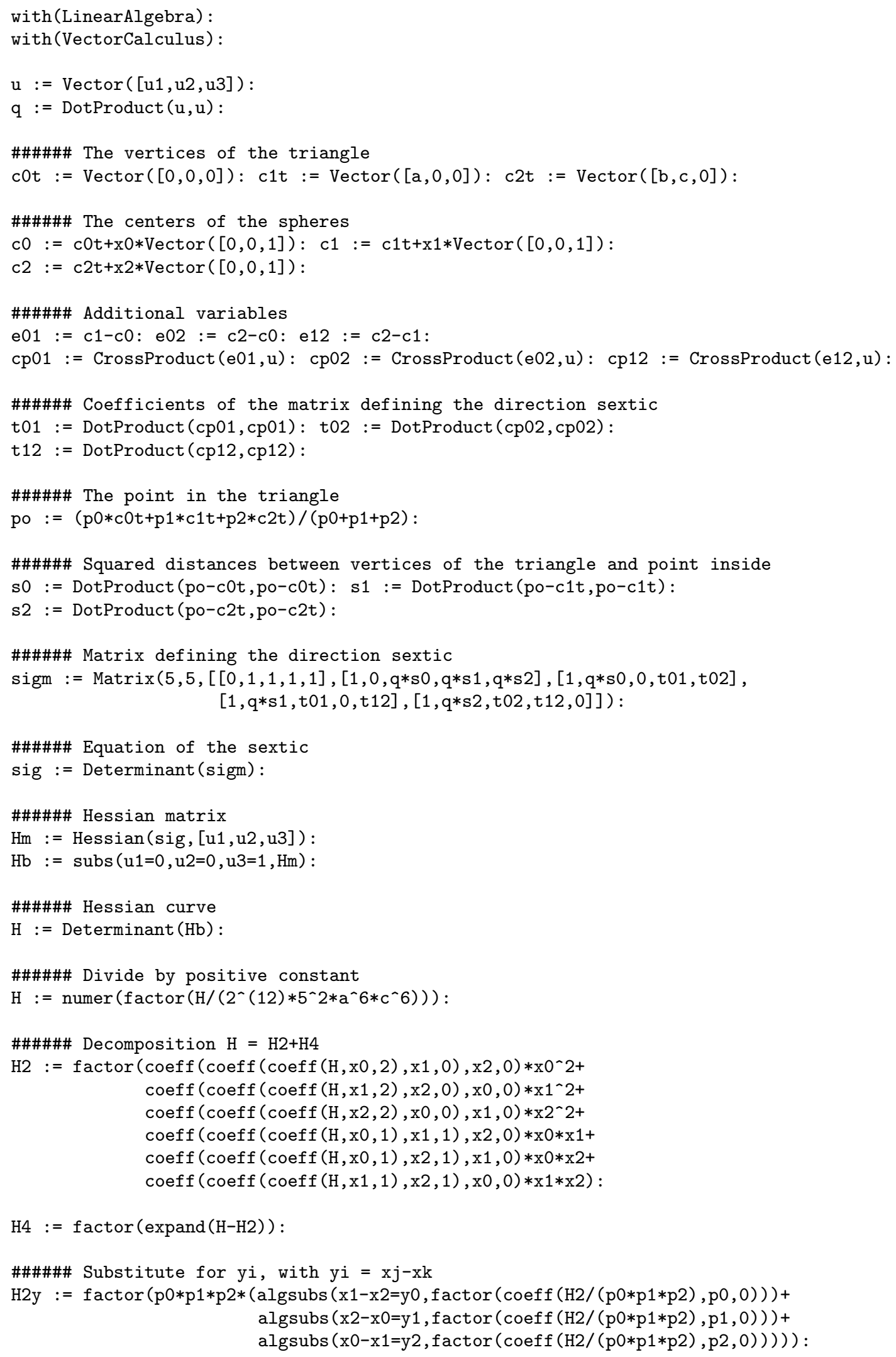


$\mathrm{H} 4 \mathrm{y}:=\operatorname{algsubs}\left(\right.$ numer $(\mathrm{s} 0 \mathrm{t})=\mathrm{r} 0^{\wedge} 2$, algsubs $(\mathrm{x} 2-\mathrm{x} 0=\mathrm{y} 1, \operatorname{algsubs}(\mathrm{x} 0-\mathrm{x} 1=\mathrm{y} 2$,

factor (coeff $(\mathrm{H} 4, \mathrm{p} 0,3))$, exact), exact), exact) $* \mathrm{p} 0 \wedge 3+$

algsubs (numer $(\mathrm{s} 1 \mathrm{t})=\mathrm{r} 1^{\wedge} 2$, algsubs $(\mathrm{x} 1-\mathrm{x} 2=\mathrm{y} 0, \operatorname{algsubs}(\mathrm{x} 0-\mathrm{x} 1=\mathrm{y} 2$,

factor (coeff $(H 4, p 1,3))$, exact), exact), exact) *p1^3+

algsubs (numer $(s 2 t)=r 2 \uparrow 2, \operatorname{algsubs}(x 1-x 2=y 0, \operatorname{algsubs}(x 2-x 0=y 1$,

factor ( $\operatorname{coeff}(\mathrm{H} 4, \mathrm{p} 2,3))$, exact), exact $)$, exact $) * \mathrm{p} 2 \uparrow 3$ :

\#\#\#\#\# Substitute $\mathrm{qk}=\mathrm{pk} * \mathrm{rk}$ and $\mathrm{zk}=\mathrm{yk}^{\wedge} 2$

$\mathrm{H} 2 \mathrm{z}:=\operatorname{algsubs}\left(\mathrm{y}^{\wedge} 2=\mathrm{z} 0\right.$, $\left.\operatorname{algsubs}\left(\mathrm{y} 1^{\wedge} 2=\mathrm{z} 1, \operatorname{algsubs}\left(\mathrm{y} 2^{\wedge} 2=\mathrm{z} 2, \mathrm{H} 2 \mathrm{y}\right)\right)\right)$ :

$\mathrm{H} 2 \mathrm{z}:=\operatorname{algsubs}(\mathrm{p} 0 * r 0=\mathrm{q} 0, \operatorname{algsubs}(\mathrm{p} 1 * r 1=\mathrm{q} 1, \operatorname{algsubs}(\mathrm{p} 2 * r 2=\mathrm{q} 2, \mathrm{H} 2 \mathrm{z})))$ :

$\mathrm{H} 4 \mathrm{z}:=\operatorname{algsubs}\left(\mathrm{y} 0^{\wedge} 2=\mathrm{z} 0, \operatorname{algsubs}\left(\mathrm{y} 1^{\wedge} 2=z 1, \operatorname{algsubs}\left(\mathrm{y} 2^{\wedge} 2=z 2, \mathrm{H} 4 \mathrm{y}\right)\right)\right)$ :

$\mathrm{H} 4 \mathrm{z}:=\operatorname{algsubs}(\mathrm{p} 0 * r 0=\mathrm{q} 0, \operatorname{algsubs}(\mathrm{p} 1 * r 1=\mathrm{q} 1, \operatorname{algsubs}(\mathrm{p} 2 * r 2=\mathrm{q} 2, \mathrm{H} 4 \mathrm{z})))$ :

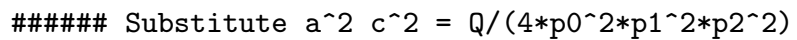

$\mathrm{H} 2 \mathrm{z}:=\operatorname{algsubs}\left(\mathrm{a}^{\wedge} 2 * \mathrm{c}^{\wedge} 2=\mathrm{Q} /\left(4 * \mathrm{p} 0^{\wedge} 2 * \mathrm{p} 11^{\wedge} 2 * \mathrm{p} 2{ }^{\wedge} 2\right), \mathrm{H} 2 \mathrm{z}\right)$ :

\#\#\#\#\# Substitute pi*pj*zk $=\mathrm{qk} \wedge 2 * w k$ and multiply by a positive scalar

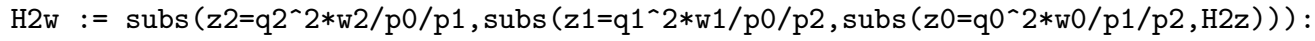

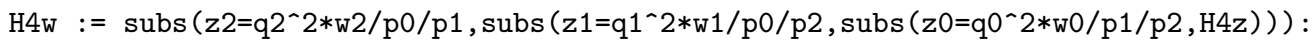

$\mathrm{H} 2 \mathrm{w}:=$ factor $(4 * \mathrm{p} 0 * \mathrm{p} 1 * \mathrm{p} 2 * \mathrm{H} 2 \mathrm{w})$ :

$\mathrm{H} 4 \mathrm{w}:=$ factor $(4 * \mathrm{p} 0 * \mathrm{p} 1 * \mathrm{p} 2 * \mathrm{H} 4 \mathrm{w}):$

\#\#\#\#\# The vertex $\mathrm{V}$ of the disjointness conditions

v0 := 1-(q1-q2)^2/q0^2: v1 $:=1-(\mathrm{q} 2-\mathrm{q} 0)^{\wedge} 2 / \mathrm{q}^{\wedge} 2:$ v2 $:=1-(\mathrm{q} 0-\mathrm{q} 1)^{\wedge} 2 / \mathrm{q} 2^{\wedge} 2$ :

\#\#\#\#\# Evaluation of $\mathrm{H}$ at this vertex

print (factor (subs ( $\mathrm{w} 0=\mathrm{v} 0$, $\mathrm{w} 1=\mathrm{v} 1$, $\mathrm{w} 2=\mathrm{v} 2$,



\#\#\#\#\# Evaluation of the plane t0+t1+t2 at the vertex $\mathrm{V}$ for $\mathrm{q} 0=\mathrm{q} 1=\mathrm{q} 2$

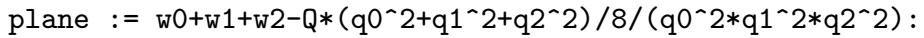

print (subs ( $11=\mathrm{q} 0, \mathrm{q} 2=\mathrm{q} 0, \operatorname{subs}(\mathrm{w} 0=\mathrm{v} 0, \mathrm{w} 1=\mathrm{v} 1, \mathrm{w} 2=\mathrm{v} 2$,

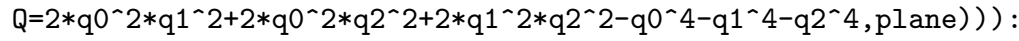

